\title{
Quality of Service in WSN-A Review
}

\author{
Sukhkirandeep Kaur \\ Student, CSE Deptt. \\ National Institute of Technology \\ Srinagar
}

\author{
Roohie Naaz Mir \\ Professor, CSE Deptt. \\ National Institute of Technology \\ Srinagar
}

\begin{abstract}
The capability of sensor nodes to observe and react to physical phenomenon has made wireless sensor networks a new technological vision. They are small devices having distributed sensing capabilities with wireless interfaces and communicate using Radio Communication (RF) techniques. As these networks are used for applications that emphasize on timeliness or reliability or both QoS becomes an important parameter. But due to resource constrained and power hungry nature of these devices very few work is done in this area and there is currently no standardization, on a framework and/or general guidelines in the networking community on how QoS can be achieved in WSNs. This paper presents the Quality of service parameters, issues and about the work done in this area.
\end{abstract}

\section{General Terms}

Wireless Sensor Networks, Quality of Service, Routing

\section{Keywords}

Wireless Sensor Networks, Quality of Service, Timeliness, Reliability, End to end delay

\section{INTRODUCTION}

Wireless Sensor Networks has been identified as most important technology for low power wireless communication. The ability of small, low cost devices called sensor nodes to cooperatively monitor physical or environmental conditions such as pressure, humidity, temperature has increased their importance. Visual Sensor Networks that involves cameras are also emerging form of WSN for real time applications. WSN have wide range of applications in military, environmental monitoring, healthcare, habitat monitoring and surveillance. But limited memory, low power and limited processing nature of WSN impose several issues also. Energy conservation is an important issue to be considered. WSN are also used in real time applications, the transmission of imaging and video data requires careful handling in order to ensure that end-to-end delay is within the acceptable range and that the variation in such delays is acceptable [1] so reliability and timeliness becomes important QoS parameters in real time applications. QoS has become very important topic in WSN research.

Different QoS parameters were used in traditional networks such as packet loss, delay, jitter, bandwidth etc. However, the QoS requirements in WSNs such as data accuracy, aggregation delay, coverage, fault tolerance and network lifetime etc. are application specific and they are different from the traditional end-to-end QoS requirements due to the difference in application domains and network properties [2]. The QoS models used in internet and adhoc networks like Differentiated Services (DiffServ) and Integrated Services (IntServ) cannot be used in WSN due to their different nature.

In case of WSN QoS is provided by layered and cross layered approach. Layered approach deals with specific layer and cross layer requires simultaneous interaction of different layers. Both layers provide required QoS to users and application but at the cost of reduced energy. QoS is an umbrella term for a collection of technologies that allow network-aware applications to request and receive predictable service levels in term of QoS requirements [3].

The rest of the paper is organized as follows. In Section 2 we have discussed the characteristics of WSN which pose challenges for QoS support. Section 3 discusses the classification of QoS in WSN. In Section 4 detailed survey of the existing approaches for QoS support in WSN is done and some open research issues for QoS support in WSNs are listed in Section 5.

\section{CHALLENGES FOR QOS SUPPORT IN WSN}

Nature of WSN is very different from adhoc networks and internet. So the requirements with respect to these networks are also different. Moreover resource constraint and power hungry nature of WSN impose several challenges also which are discussed below

- Resource Constraints: As we know sensor nodes are deployed in hostile environment and the battery can be drained quickly and there is no source to replace or recharge the battery so energy becomes an important constraint. Other constraints are memory, limited processing and transmission power.

- Scalability: Depending upon the application requirements sensor nodes can increase therefore QoS should support scalability. Performance of network should not be degraded as number of nodes increases.

- Fault tolerance: Due to depletion of energy node can fail. In case of node failures network should cope up as soon as possible but network dynamics such as node failures, link failures imposes challenges for QoS.

- Packet Criticality: Depending upon the application criticality of packet is identified and priority is assigned. For real time packets higher priority is assigned as they are delay bounded. QoS mechanisms should differentiate packet importance and set up priority structure.

- Multiple sinks and traffic types: Depending upon the application requirements multiple sinks and sensors may be involved for different purposes. This makes QoS more challenging.

- Real time requirements: Multimedia applications and Performance critical applications have different delay requirements. Hard real time delay guarantees become difficult to achieve because of the limited resource and energy issues of WSN. Multimedia data requires high bandwidth and low delay. So 
predefined QoS is required in these applications to achieve required service.

\section{QoS IN WSN}

QoS in WSN can be application specific or network specific.WSN is application specific, for some application like forest monitoring delay is a very important factor. Information regarding any harmful event should reach within fraction of seconds. For applications involving object tracking due to improper coverage of any sensor or lack of active sensors target can be missed. So in this type of applications we can define coverage or active sensors as parameters to measure QoS in WSN. And for multimedia applications involving camera and video sensors real time delivery of data is important. Depending upon the application different QoS requirements can be imposed on WSNs. Different application specific parameters are coverage, optimum number of active sensors, fault tolerance, data accuracy and aggregation delay. At application layer as whole different parameters which are considered are System lifetime, Response time, Data reliability etc.

Network QoS specifies how underlying communication network can deliver QoS constrained data while efficiently utilizing network resources. Basically network QoS deals with the delivery of data and there are different data delivery models i.e. Continuous, Query Driven and Event Driven.

1) Continuous Data delivery model sends the data periodically to real and non real time applications. Real time applications are delay intolerant but in case of non real time delay can be tolerated to a certain extent.

2) In Query Driven applications query is generated by sink and sensor nodes respond to that query. These are used in mission critical applications and Delay tolerance is query specific. Reliability is of main concern in these applications. Query driven is also used to manage the sensor nodes. If there is any change that is to be made to sensor node, sink can send out command to execute these changes.

3) In case of Event Driven Applications whenever sensor nodes observe any event, that event should be reported to sink in timely fashion and appropriate action should be taken depending upon the event. So these applications are mission critical and delay intolerant. This differs from Query driven as in case of event driven data is pushed to the sink and in query driven data is pulled by the sink.

At the network layer Path latency, Routing maintenance, Congestion probability and Energy efficiency are the main QoS parameters which are considered. Various techniques used to achieve QoS in network layer are:

- Multipath Routing: Multiple copies of each packet are sending over multiple paths to improve reliability, load balancing, security and to achieve QoS.

- Data aggregation: In network data aggregation reduces amount of data to be sent over the network. As multimedia applications require huge amount of data to be sent and due to bandwidth limitations aggregation is done to reduce redundancy and to utilize bandwidth.
- Energy aware routing: Energy efficient path is found that meets end to end delay requirements.

- Minimum cost forwarding: Minimum cost path in terms of bandwidth, energy is found. So depending upon the Data delivery model different QoS requirements can be imposed on the network

\section{QoS AWARE ROUTING PROTOCOLS}

QoS routing protocols can be differentiated based on timeliness or reliability. Timeliness is needed in applications which have severe delay requirements and reliability is needed in cases where data is of foremost importance. For measuring QoS important parameters which are considered are Throughput, jitter, Delay and Packet loss. A lot of work has been done and still going on in improving QoS in WSN. Energy Conservation is also considered as an important parameter. Many protocols have been developed and evaluated on the basis of above parameters. Some of them are listed below

\subsection{Sequential Assignment Routing (SAR)}

SAR is the first routing protocol that included notion of QoS. Both energy efficiency and fault tolerance are included in this protocol. It is a multi path, table driven routing protocol which creates a tree of sensor nodes having root at the one hop neighbour of the sink node. Paths are established by taking into account the QoS metrics, energy resource in each path and priority of each packet. Based on QoS and energy resource of each path multiple paths are selected. In case of large number of sensor nodes overhead increases as large number of nodes are employed.

\subsection{SPEED}

SPEED is a localized protocol which is used when node density is high and resources are scarce. Information about nodes is exchanged through neighbour beacon exchange message and geographic forwarding is used to forward messages. Delay is calculated via Receive Delay Estimator and next hop is chosen based on Stateless Non Deterministic Geographic forwarding, forwarding candidate set of nodes is chosen and relay speed is calculated by dividing the distance by hop delay. Packets are forwarded to nodes belongs to forwarding set, if no such node is found packet are dropped Nodes whose relay speed is higher than Ssetpoint, they are chosen as next hop. If no such node is found then neighbor feedback loop is used. Limitation of this protocol is that it provides in network wide speed which is not suitable for differentiating various traffic with different deadlines. Same preference is given to both real and non real time traffic. It does not consider any energy and reliability metric.

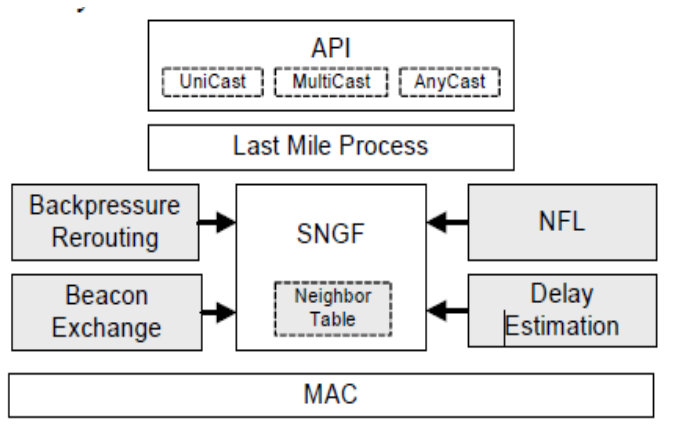

Figure1. Basic architecture of SPEED [6] 


\subsection{MMSPEED}

Multi-Path and Multi-SPEED Routing is a multipath multispeed protocol that provides real time guarantee. It is an extension to SPEED Protocol QoS is provided in two domains, reliability and timeliness. By guarantying multiple packet delivery speed options timeliness is provided and reliability is provided by multipath forwarding. When an event is detected packet is created to be transmitted. Based on content of packet appropriate end to end deadline and required reaching probability is found. Then packet forwarding is done by MMSPEED protocol. Packets are divided into different speed layers according to their priorities and their scheduling is done accordingly.

Reliability is provided by sending high reliable packet over multiple paths. MMSPEED is used only for short lived applications and it does not consider energy efficiency at all [7].

\subsection{QEMPAR}

QEMPAR is QoS based protocol which also considers energy consumption as an important parameter. It is a location based protocol and it is assumed that nodes know their location, their remaining energy and remaining energy of other nodes in their transmission range. Energy model use both open space and multipath channels by taking the distance between transmitter and receiver. Then link suitability is found by using PPS (Probability of packet sending) and APPR (Average probability of packet receiving) and $\mathrm{I}_{B}$ (Interference of link A and B). Then node disjoint paths are found. After path discovery Real time packets are broken into smaller packets and are sent over consecutive paths. Results are compared with MCMP protocol and it shows better performance in terms of End to End delay and average energy consumption.

\subsection{EAQoS}

Energy Aware QoS routing protocol[5] is QoS aware protocol for real time traffic. It also maximize throughput for non real time traffic. Best delay constrained path based on cost function is found from multiple paths. Optimal path is found considering energy for real time consumption and error rate while considering end to end delay required for real time data. Queuing model is employed which divides the traffic into Real Time and Non-Real Time and allocates bandwidth according to value of $r$ (Bandwidth ratio). Paths are found and Dijkastra's algorithm paths are arranged according to their least cost. R.

\subsection{EQSR}

Energy efficient and QoS based routing protocol (EQSR) is multipath routing protocol which considers reliability, energy efficiency and delay as important QoS parameters and also includes XoR based FEC technique. Next hop is selected based on residual energy, available buffer size and Signal to noise ratio. Message is splitted into segments; correction code is added and is transmitted over multiple paths. Service differentiation is provided by using Queuing model which handles both real and non real time traffic.

\subsection{RTLLR}

Real-time link-Reliability routing is QoS based protocol which considers both reliability and timeliness by considering link reliability, Delay and energy efficiency. It considers two hop neighborhood information for making routing decisions. It mainly focuses on two parameters Link Reliability and Link delay and based on that information next hop is selected. In RTLRR if a link with higher PRR is selected probability of successful packet delivery to the forwarding node is increased. It is compared with SPEED and THVR and shows better performance in terms of delay and energy consumption.

\subsection{RRQRP}

Reliable and Robust QoS routing protocol for WSNs based on a Combined Weight $(\mathrm{CW})$ Value. The $\mathrm{CW}$ is based on the QoS parameters link quality, residual energy and available bandwidth. To estimate Link Quality node mobility and RSSI are considered. Available bandwidth is estimated from

$$
\mathrm{AW}_{\mathrm{BW}}=\operatorname{Max}_{\mathrm{BW}} *\left(\frac{\text { Idle }_{\mathrm{t}}}{\text { Int }_{\mathrm{t}}}\right)
$$

Residual energy is calculated by subtracting energy consumed by the node from initial energy and link quality is estimated from SNR. If $\mathrm{CW}$ value is higher than $\mathrm{MCW}$ (minimum combined weight value) then all copies of route request are forwarded otherwise negative acknowledgement is sent. It shows better performance as compared to QBRP in terms of energy, delay.

\subsection{QEMH}

QoS Aware and Multi-path Hierarchical protocol is energy aware clustering based protocol that gives preference to real time traffic over non real time so that real time data gets delivered with a very little delay. Cluster head is chosen based on residual energy and node Distance to sink. Every node broadcast these two parameter values and node having maximum value is chosen as cluster head. After selecting cluster head, next hop is chosen by cluster head based on energy factor, available buffer and link performance factor. RREQ message is sent to next preferred node until sink is reached. Alternative paths are found in this way and path having lower delay is chosen for real time traffic. To improve reliability correction codes are added using XoR based correction methods. Comparison is done with MCMP and EAP protocols and it achieves more energy saving, low average end to end delay, more lifetime and high packet delivery ratio. 
Table 1: Comparison of different Protocols

\begin{tabular}{|lcccc|}
\hline Routing Protocol & Multi-path & Energy efficiency & Reliability & QoS \\
\hline SAR & YES & HIGH & - & YES \\
SPEED & YES & LOW & LOW & YES \\
MMSPEED & YES & LOW & HIGH & YES \\
QEMPAR & YES & HIGH & HIGH & YES \\
EAQoS & YES & HIGH & HIGH & YES \\
EQSR & YES & HIGH & HIGH & YES \\
RTLLR & NO & HIGH & HIGH & YES \\
RRQRP & YES & HIGH & LOW & YES \\
QEMH & YES & HIGH & HIGH & YES \\
\hline
\end{tabular}

\section{RESEARCH ISSUES}

As already discussed WSN are very different from adhoc networks so Diffserv and Interserv models cannot be used in WSN. To achieve QoS simple models should be used using cross layer approaches. Mobility is the main issue to be considered as most of the time it is assumed that sensor node and sink are stationary but there exist certain scenarios, for example, in military environment, the sensor nodes and the sink will be made mobile. So efficient techniques for QoS considering mobility should be developed. Also, the topology of the network may also keep on dynamically changing. Therefore, efficient routing protocols are required to address mobility and dynamicity of the wireless sensor network [2]. In case of real time applications Reliability and timeliness is an important concern in providing QoS. Data redundancy may exploit reliability; if we use fusion techniques it will effect timeliness and introduces delay. So optimum techniques should be developed to overcome this issue. Multipath techniques should be developed to ensure delivery of data in timely and reliable fashion. Moreover different cross layer techniques should be developed and different QoS control mechanisms should be developed to achieve Quality of Service in WSN. Most importantly the major issue of WSN i.e. Energy consumption should be considered while developing new protocols and techniques.

\section{CONCLUSION}

So far very few work and effort has been done in field of QoS in WSN. Due to the development of both real time as well as non real time application providing QoS is a very important and major issue to be considered. Energy efficiency is the main issue to be considered while developing protocols and algorithms. Moreover to meet timeliness and reliability demand of WSN application different QoS techniques like multipath routing to increase reliability, Queuing model to differentiate real and non real time traffic, clustering techniques to overcome problems of scalability should be used.

In this paper we have discussed challenges imposed by several characteristics and discussed useful parameters of providing QoS in WSN. We have discussed several routing protocols that include the notion of QoS and compared them on basis of different parameters. Different issues related to QoS have been identified and discussed.

\section{REFERENCES}

[1] Sumathi,R. and Srinivas,M.G. . A Survey of QoS Based Routing Protocols for Wireless Sensor Networks, Vol.8, No.4, December 2012

[2] Bhuyan,B., Kumar,H., Sarma, D., Sarma,N. Quality of Service (QoS) Provisions in Wireless Sensor Networks and Related Challenges. Wireless Sensor Network, 2010, $2,861-868$

[3] Balen,J., Zagar,D., and Martinovic,G. Quality of Service in Wireless Sensor Networks: A Survey and Related Patents, ISSN (Online): 1874-4796

[4] Ganz,A., Ganz, Z. and Wongthavarawat, K. 2004. Multimedia Wireless Networks: Technologies, Standards, and $Q o S$, Prentice Hall, Upper Saddle, NJ

[5] K. Akkaya and M. Younis, "An Energy-Aware QoS Routing Protocol for Wireless Sensor Networks," Proc. of the IEEE Workshop on Mobile and Wireless Networks (MWN 2003), Providence, RI, May 2003.

[6] Tian He, John A Stankovic, Chenyang Lu, Tarek Abdelzaher," SPEED: A Stateless Protocol for RealTime Communication in Sensor Networks," in proceedings of 23rd International Conference on Distributed Computing Systems, May 2003.

[7] Emad Felemban, Chang-Gun Lee, Eylem Ekici," MMSPEED: Multipath Multi-Speed Protocol for QoS of $\mathrm{R}$ Reliability and Timeliness in Wireless Sensor Networks," in IEEE Transactions on Mobile Computing, volume: 5, 2006.

[8] Shiva Prakash T, Raja K B,2014. Real-Time Link Reliability Routing for QoS in Wireless Sensor Networks. e-ISSN: 2278-0661, PP 05-16.

[9] D. Chen and P. K. Varshney, "QoS Support in Wireless Sensor Network A Survey," Proceedings of the 2004 Sensor Network: International Conference on Wireless Sensor Networks (ICWN2004), Las Vegas, Nevada, USA,June 2004.

[10] Chimkode, S.,Ramesh S. Jadhav. 2012. A Reliable and Robust QoS routing protocol for WSNs, International Journal of Computer Applications (0975-888), and Journal Volume 47-No.14. 
[11] E. Crawley, R. Nair, B. Rajagopalan and H. Sandick, 1998. A Framework for QoS-Based Routing in the Internet, RFC2386

[12] Iyer,R. and Kleinrock,L.2003 QoS Control for Sensor Networks Vol. 1, 11-15 May 2003, pp. 517-52

[13] J.B Othman, B. Yahya, "Energy efficient and QoS based Routing protocol for wireless sensor networks" , Journal of Parallel and Distributed Computing, vol. 70 Issue 8, pp. 849-857, August 2010

[14] Martínez, J., García, A., Corredor, I., López, L., Hernández. V., and Dasilva, A. 2007. QoS in wireless sensor networks: Survey and approach. EATIS.
[15] Sohrabi, K., Goa, J., Ailawadhi, V., and Pottie, G. J. 2000. Protocols for self-organization of a wireless sensor network. IEEE Personal Commun 7, 5 (Oct.), $16--27$.

[16] Mohammad R. Mazaheri, Homayounfar, B.,Mazinani, S,M 2012, Wireless Sensor Network, 2012, 4, 31-39.

[17] X. Huang and Y. Fang, "Multiconstrained QoS Mutlipath Routing in Wireless Sensor Networks," Wireless Networks, Vol. 14, No. 4, 2008, pp. 465-478. 